\title{
Stroke and Sleep Apnoea: Cause or Consequence?
}

\section{Bhatia}

Indian J Sleep Med 2006; 1.1, 19-21

See end of article for authors

affiliations

Address for correspondence:

Dr. Manvir Bhatia

L-23, Hauz Khas Enclave,

New Delhi - 110016

manvirbhatia1@yahoo.com

\author{
Keywords: OSA, STRO KE, CPAP
}

\section{Sleep Apnoea and Stroke}

Stroke is the second leading cause of death worldwide and leading cause of long-term disability., ${ }^{1,2}$ Strategies for stroke prevention, including the control of hypertension, treatment of atrial fibrillation, and smoking cessation, have reduced the disease burden, but stroke still remains an important public health challenge. $A$ better understanding of the risk factors for stroke is needed to develop additional preventive strategies. The cardiovascular implications of normal \& disturbed sleep, especially sleep apnea have recently drawn a lot of attention.

Central sleep apnea is characterized by intermittent loss of respiratory drive, resulting in apnea followed by compensatory periods of hyperventilation. O bstructive sleep apnea occurs when mechanisms that maintain upper-airway tone during sleep are dysfunctional, resulting in a narrowing or collapse of the airway. The obstructive sleep apnea syndrome is a common medical condition, a treatable form of disordered breathing in which the upper airway closes repeatedly during sleep.

\section{Prevalence of Sleep Apnoea in Stroke}

The prevalence of sleep apnea in middleaged American adult population is $4 \%{ }^{3}$ The syndrome is associated with vascular risk factors and with substantial cardiovascular morbidity and mortality. ${ }^{4}$ Several studies have shown a prevalence of the syndrome among patients with stroke that exceeds 60 percent. 5,6,7,8,9 Preval ence of SD B (AHI >5) in acute stroke ranges from $44-95 \%$, and of SDB at 3 yrs in stroke survivors (AH 15 ) is reported as $81 \%$ and (AHI 5 +ESS 11 ) being 20\%. ${ }^{10}$

Whether the relation between the syndrome and stroke is independent of confounding risk factors, such as hypertension, hyperlipidemia, diabetes mellitus, and smoking is not clear. Several cross-sectional analyses $7,11,12,13,14,15$ have shown an increase in the risk of stroke with sleep-disordered breathing that is similar in magnitude to the effect of other cardiovascular risk factors.

\section{Mechanism in Development of Cardiovascular Disease}

Although the exact cause that links OSA with cardiovascular disease is unknown, there is evidence that OSA is associated with a group of proinflammatory and prothrobotic factors that have been identified to be important in development of atherosclerosis. OSA is associated with increased daytime and nocturnal sympathetic activity. Autonomic abnormalities are also seen such as increased resting heart rate, increased BP variability. The pathophysiologic mechanisms that are seen in OSA and may participate in the development of

Indian Journal of Sleep M edicine (IJSM ), Vol. 1, N o. 1, 2006 
cardiovascular disorders are repetitive respiratory events which cause hypoxia, hypercapnia and arousals or disrupted sleep. These physiologic events result in increased sympathetic outflow, alteration in BP control mechanisms, and vascular alterations. ${ }^{16}$

Various markers such as plasma homocysteine, C reactive protein, fibrinogen, endothelin have also been implicated, along with insulin resistance. Inflammatory markers, infection, homocystiene and SD B rank as four most important new risk factors in cerebral atherosclerosis.

\section{Effect of Apnoea on Stroke}

In the immediate post stroke period areas of the brain are thought to become critically ischaemic, especially the ischaemic penumbra. So any fluctuation in cerebral blood flow or blood oxygen saturation is critical. It is well known that stroke progression typically occurs early after stroke onset, and is independently associated with SD B. ${ }^{17}$

Repeated episodes of UAO affect cerebral blood flow, cause fluctuations in intracranial pressure; upper airway occlusion causes hemodynamic oscillations in BP, cardiac output and heart rate. 18,19,20

The pharyngeal muscles may be affected in stroke, thus causing UAO. Systemic B.P., pulse rate and cardiac output all decrease during an obstructive apnoea and increase suddenly at apnoea termination. Cerebral blood flow also decreases with upper airway obstruction. $43 \%$ of stroke patients have progression of their neurological deficit. This typically occurs early after stroke onset, with $87 \%$ occurring within first 48 hours.

W hy the interest? If UAO is common during sleep in first 24 hours of stroke; interventions targeted to abolish sleep disordered breathing may improve outcome.

\section{Effect of OSA on morbidity and Mortality Post Stroke}

The fact that UAO occurring with 24 hours of stroke affects the prognosis is not surprising, given the haemodynamic oscillations and fluctuations in oxygen saturation that accompany it.

It has been seen that severity of upper airway obstruction appears to be associated with a worse functional outcome following stroke, increasing the likelihood of death. L onger respiratory events had greater effect (mean length of respiratory event most significantly associated with death at 6 mths was 15 secs. ${ }^{21}$ Various authors evaluated SD B \& stroke, and have reported worse functional outcome in those with SDB.

Good et al ${ }^{22}$ studied patients with pulse oximetry, and found that a number oximetry variables (desaturation index, mean $\mathrm{SaO} 2$, no. of desaturations) correlated with the Barthel index at discharge and at $3 \& 12$ months. Spriggs et al ${ }^{12}$ reported 6 mth survival for stroke patients as $79 \%$ for those who "never snored" compared with $52 \%$ who "always snored". The early and late mortality rates in non-regular snorers (4 -18\%) was lower than in regular snorers. (15\% $-30 \%)$.

ThusU AO is associated with mortality and morbidity following stroke; however because of unsuspected and unmeasured confoundersinherent in observational study, this cannot be taken as confirming causation.

A recent study showed that severe obstructive sleep apnoea syndrome increases the risk of fatal and nonfatal cardiovascular events and that this isindependent of other cardiovascular and cerebrovascular risk factors. The risk of stroke increased progressively with increased severity of the syndrome. ${ }^{23}$ An observational study has reported that OSAS significantly increases the risk of stroke or death from any cause and the increase is independent of other risk factors, including hypertension. ${ }^{24}$

\section{CPAP after Stroke}

CPAP compliance is $50-70 \%$ in highly selected stroke patients in stable phase following stroke. The questions about CPAP such as fixed pressure, auto titration and at what pressure remain unanswered.

\section{Condusion}

The interrelation between snoring, sleep apnoea and stroke remains a challenge. Potentially the topic is of considerable importance as risk factors for stroke and management of patients after stroke.

Even if it is not possible to eliminate all obstructive respiratory events, at least preventing longer events, and hence more severe hemodynamic oscillations with oxygen desaturation may be effective in improving outcome.

The questions whether OSA is an independent risk 
factor for stroke?

Probably answered by NEJM study

Can treatment of OSA reduce the risk of stroke?

Increased prevalence/severity of OS A post stroke? Yes

$D$ oes sleep apnoea independently influence the mortality for stroke? Possibly

\section{Effect on recovery - ?}

However, although the use of CPAP in obstructive sleep apnea may lower blood pressure, sympathetic activity, and other surrogates of cardiovascular risk, there are no large-scale, randomized trials of cardiovascular events or survival with the treatment. Although therapy should always be tailored to the individual patient, the intriguing data from Bradley, Yaggi, and their colleagues provide a timely reminder of the importance of evidence based recommendations in any widespread therapeutic strategy, particularly when treatment options carry a substantial economic burden.

\section{Authors' affiliations}

Dr. Manvir Bhatia, Senior Consultant in N eurology, Sir Ganga Ram Hospital, New Delhi

\section{Reference}

1. Murray CJ, Lopez AD. Mortality by cause for eight regions of the world: Global Burden of Disease Study. Lancet 1997; 349: 1269-76.

2. Heart disease and stroke statistics-2005 update. Dallas: American Heart Association, 2005.

3. Young T, Palta M, Dempsey J, Skatrud J, Weber S, Badr S. The occurrence of sleep-disordered breathing among middle-aged adults. N Engl J Med 1993; 328: 12301235.

4. Young T, Peppard PE, Gottlieb DJ. Epidemiology of obstructive sleep apnea: a population health perspective. Am J Respir CritCare Med 2002; 165:1217-1239.

5. Mohsenin V, Valor R. Sleep apnea in patients with hemispheric stroke. Arch Phys Med Rehabil 1995; 76: 71-76

6. Dyken ME, Somers VK, Yamada T, Ren ZY, Zimmerman MB. Investigating the relationship between stroke and obstructive sleep apnea. Stroke 1996; 27:401-407,

7. Bassetri C, Aldrich MS. Sleep apnea in acute cerebrovascular diseases: final report on 128 patients. Sleep 1999; 22:217- 223

8. Parra O, Arboix A, Bechich S, et al. Time course of sleeprelated breathing disorders in first-ever stroke or transien ischemic attack. Am J Respir Crit Care Med 2000; 161: 375-380.
9. Turkington PM, Bomford J, Wanklyn $\mathrm{P}$, et al. Prevalence and predictors of upper airway obstruction in the first 24 hours after acute stroke. Stroke 2002; 33: 2037- 2042.

10. Cadilhac DA, Thorpe RD, Pearce DC, Barnes M, Rochford PD, Tarquinio N, Davis SM, Donnan GA, Pierce RJ. Sleep disordered breathing in chronic stroke survivors. A study of the long term follow-up of the SCOPES chort using home based polysomnography. J Clin Neuro Sci 2005; 12(6): 632-637.

11. Partinen $\mathbf{M}$, Palomaki H. Snoring and cerebral infarction. Lancet 1985; 2: 1325-1326.

12. Spriggs DA, French JM, Murdy JM, Curless RH, Bates D, James OF. Snoring increases the risk of stroke and adversely affects prognosis. QJ Med 1992; 83: 555-562.

13. Smirne S, Palazzi S, Zucconi M, Chierchia S, Ferini-Strambi L. Habitual snoring as a risk factor for acute vascular disease. Eur Respir J 1993; 6:1357-1361.

14. Neau JP, Meurice JC, Paquereau J, Chavagnat J, Ingrand P, Gil R. Habitual snoring as a risk factor for brain infarction. Acta Neurol Scand 1995; 92: 63-68

15. Shahar E, Whitney CW, Redline S, et al. Sleep-disordered breathing and cardiovascular disease: cross-sectional results of the Sleep Heart Health Study. Am J Respir Crit Care Med 2001; 163:19-25.

16. Foresman BH, Gwirtz PA, McMahon JP. Cardiovascular disease and obstructive sleep apnea: implications for physicians. J Am Osteopath Assoc 2000 June; 100 (6); 360-369.

17. Iranzo A, Santamaria J, Berenguer J, et al. Prevalence and clinical importance of sleep apnea in the first night after cerebral infarction. Neurology 2002; 58: 911-916.

18. Guilleminault C, Connolly SJ, Winkle RA. Cardiac arrhythmia and conduction disturbances during sleep in 400 patients with sleep apnoea syndrome. Am J Cardiol 1983; 82: 490 - 494.

19. Fischer A, Choudhary B, Taormina M, et al. Intracranial hemodynamics in sleep apnea. Chest 1992; 102:14021406, Netzer N, Wermer P, Jochums I, et al. Blood flow of the middle cerebral antery with sleep-disordered breathing. Stroke 1998; 29:87-93.

20. Hayakawa T, Terashima M, Kayukawa $Y$, et al. Changes in cerebral oxygenation and hoemodynamics during sleep apnoeas. Chest 1996; 109:916- 921.

21. Turkington PM, Allgar V, Bamford J, Wanklyn P, Elliott MW. Effect of upper airway obstruction in acute stroke on functional outcome at 6 months. Thorax 2004; 59: 367371.

22. Good D, Henkle J, Gelber D, et al. Sleep disordered breathing and poor functional outcome after stroke. Stroke 1996; 27:252-259.

23. Marin J M, Carrizo SJ, Vincente E, Agusti AG. Long-term cardiovascular outcomes in men with obstructive sleep apnoea-hypopnoea with or without treatment with continuous positive airway pressure: an observational study. Lancet 2005; 365:1046-1053.

24. Yaggi HK, Concato J, Kernan WN, Lichtman JH, Brass LM, Mohsenin V. O bstructive sleep apnea as a Risk-Factor for stroke and death. N Engl J Med 2005; 353:2034-2041.

Indian Journal of Sleep M edicine (IJSM ), Vol. 1, N o. 1, 2006 